\title{
Real-Time Welfare-Maximizing Regulation Allocation in Aggregator-EVs Systems
}

\author{
Sun Sun*, Min Dong ${ }^{\dagger}$, and Ben Liang* \\ *Dept. of Electrical and Computer Engineering, University of Toronto, Canada \\ ${ }^{\dagger}$ Dept. of Electrical, Computer and Software Engineering, University of Ontario Institute of Technology, Canada \\ Email: \{ssun, liang\}@comm.utoronto.ca, min.dong@uoit.ca
}

\begin{abstract}
The concept of vehicle-to-grid (V2G) has gained recent interest as more and more electric vehicles (EVs) are put to use. In this paper, we consider a dynamic aggregator-EVs system, where an aggregator centrally coordinates a large number of EVs to perform regulation service. We propose a Welfare-Maximizing Regulation Allocation (WMRA) algorithm for the aggregator to fairly allocate the regulation amount among the EVs. The algorithm operates in real time and does not require any prior knowledge on the statistical information of the system. Compared with previous works, WMRA accommodates a wide spectrum of vital system characteristics, including limited EV battery size, EV self charging/discharging, EV battery degradation cost, and the cost of using external energy sources. Furthermore, our simulation results indicate that WMRA can substantially outperform a suboptimal greedy algorithm.
\end{abstract}

\section{INTRODUCTION}

Electrification of personal transportation is expected to become prevalent in the near future. For example, millions of electric vehicles (EVs) will be operated in the United States by 2015 [1]. Besides serving the purpose of transportation, EVs can also be used as distributed electricity generation/storage devices when plugged-in [2]. Hence, the concept of vehicleto-grid (V2G), referring to the integration of EVs with the power grid, has received increasing attention [2]-[4].

Frequency regulation is a service to maintain the balance between power generation and load demand, so as to stabilize the frequency and voltage in a power grid. Traditionally, regulation service is achieved by turning on or off fast responsive generators and is the most expensive ancillary service [5]. Since EV power electronics and battery can well respond to the regulation signal, it is possible for plugged-in EVs to provide regulation service [6]. However, since the regulation service is generally requested on the order of megawatts (MWs) and the power capacity of an EV is typically $5-20 \mathrm{~kW}$, it is often necessary for an aggregator to coordinate a large number of EVs to provide regulation service [7].

There is a growing body of recent works on $\mathrm{V} 2 \mathrm{G}$ regulation service. Specific to the aggregator-EVs system, which focuses on the interaction between the aggregator and EVs, centralized regulation allocation is studied in [8]-[12], where the objective is to maximize the profit of the aggregator or the EVs. For example, in [8], a set of schemes based on different criteria of fairness among EVs are provided. In [9], the regulation allocation problem is formulated as quadratic programming. In [10], considering both regulation service and spinning reserves, the underlying problem is formulated as linear programming. In [11], the charging behavior of EVs is also considered, so that the problem is then reduced to the control of the charging sequence and charging rate of each $\mathrm{EV}$, which is solved by dynamic programming. In [12], a real-time regulation control algorithm is proposed by formulating the problem as a Markov decision process, with the action space consisting of charging, discharging, and regulation. Finally, a distributed regulation allocation system is proposed in [13] using game theory, and a smart pricing policy is developed to incentivize EVs.

However, these earlier works have omitted to consider some essential characteristics of the aggregator-EVs system. For example, deterministic models are used in [8] and [11], which ignore the uncertainty in the system. The dynamics of the system is not incorporated in [13], nor the energy restrictions of EV battery is considered. The self charging/discharging activities in support of the EV own needs are omitted in [8] and [13]. The cost of EV battery degradation is not considered in [9], [11]-[13], and the cost of using external energy is ignored in [8]-[12].

In this paper, we consider all of the above factors in a more complete aggregator-EVs system model, to develop a real-time algorithm for the aggregator to fairly allocate the regulation amount among the EVs. We aim to maximize the long-term social welfare of the aggregator-EVs system, under the long-term constraint on the battery degradation cost of each EV. We demonstrate how a solution to this maximization can be formulated under a general Lyapunov optimization framework [14] and propose dynamic allocation strategies specific to the aggregator-EVs system. The resultant WelfareMaximizing Regulation Allocation (WMRA) algorithm does not require any statistical information of the system, and is shown to be asymptotically close to optimal as the EV battery capacity increases. Finally, WMRA is compared with a greedy algorithm through simulation and is shown to offer substantial performance gains.

The rest of this paper is organized as follows. We describe the system model and formulate the regulation allocation problem in Section II. In Section III, we propose WMRA and evaluate its performance theoretically. Simulation results are exhibited in Section IV, and we conclude in Section V.

Notation: Denote $[a]^{+}$as $\max \{a, 0\},[a, b]^{+}$as $\max \{a, b\}$, and $[a, b]^{-}$as $\min \{a, b\}$. 


\section{System Model and Problem Formulation}

In this section, we propose a centralized dynamic aggregator-EVs system and formulate the regulation allocation problem mathematically.

\section{A. Aggregator-EVs System and Regulation Service}

Consider a time-slotted system, where regulation service is performed over equal time intervals of length $\Delta t$. As an example, in New England, New York, and Ontario, the common $\Delta t$ is 5 minutes. At the beginning of each time slot $t$, the aggregator receives a random regulation signal $G_{t}$ from the power grid. If $G_{t}>0$ then the aggregator needs to perform regulation down service by absorbing $G_{t}$ units of energy from the power grid during time slot $t$, and if $G_{t}<0$ then the aggregator needs to perform regulation up service by contributing $\left|G_{t}\right|$ units of energy to the power grid during time slot $t$.

The aggregator coordinates $N$ EVs to perform regulation service and can communicate with all EVs bi-directionally. Denote $s_{i, t}$, where $0 \leq s_{i, t} \leq s_{i, \text { cap }}$, as the energy state of the $i$-th EV at the beginning of time slot $t$, where $s_{i \text {,cap }}$ represents the battery capacity of the EV. Define $\mathbf{s}_{t} \triangleq\left[s_{1, t}, \cdots, s_{N, t}\right]$. Besides providing regulation service, the EVs can charge or discharge according to their own needs independent of the aggregator. Denote such self charging and discharging energy amounts during time slot $t$ as $d_{i c, t} \geq 0$ and $d_{i d, t} \geq 0$, respectively. Due to charging/discharging circuit limitation, both quantities are upper bounded by $d_{i, \max }>0$. Assume that self charging/discharging has higher priority than regulation service and is performed at the beginning of each time slot. Then, for the $i$-th $\mathrm{EV}$, the available energy for regulation service at time slot $t$ is

$$
m_{i, t} \triangleq s_{i, t}+d_{i c, t}-d_{i d, t}=s_{i, t}+a_{i, t},
$$

where $a_{i, t} \triangleq d_{i c, t}-d_{i d, t}$. Define $\mathbf{a}_{t} \triangleq\left[a_{1, t}, \cdots, a_{N, t}\right]$. In addition, we assume that self charging and discharging cannot happen at the same time, i.e., $d_{i c, t} d_{i d, t}=0, \forall i, t$. To perform regulation service, the EVs report their initial energy states, and at each time slot report the self charging/discharging amounts (if any) to the aggregator. Then, the aggregator allocates the required regulation energy among all EVs. Denote $x_{i d, t} \geq 0$ as the amount of regulation down energy allocated to the $i$-th $\mathrm{EV}$ and $x_{i u, t} \geq 0$ as the amount of regulation up energy contributed by the $i$-th EV. Furthermore, assume that $x_{i d, t}$ and $x_{i u, t}$ are upper bounded by $x_{i, \max }>0$. Define $\mathbf{x}_{d, t} \triangleq\left[x_{1 d, t}, \cdots, x_{N d, t}\right]$ and $\mathbf{x}_{u, t} \triangleq\left[x_{1 u, t}, \cdots, x_{N u, t}\right]$. The dynamics of the $i$-th EV energy state can be expressed as

$$
s_{i, t+1}=m_{i, t}+\mathbf{1}_{d, t} x_{i d, t}-\mathbf{1}_{u, t} x_{i u, t}=s_{i, t}+a_{i, t}+b_{i, t},
$$

where $\mathbf{1}_{d, t} \triangleq\left\{\begin{array}{ll}1, & \text { if } G_{t}>0 \\ 0, & \text { otherwise }\end{array}, \mathbf{1}_{u, t} \triangleq\left\{\begin{array}{ll}1, & \text { if } G_{t}<0 \\ 0, & \text { otherwise }\end{array}\right.\right.$, and $b_{i, t} \triangleq \mathbf{1}_{d, t} x_{i d, t}-\mathbf{1}_{u, t} x_{i u, t}$. Charging a battery to near its capacity or discharging it to close to the zero energy state can significantly reduce its lifetime [15]. Therefore, lower and upper bounds for the battery energy state are usually imposed by its manufacturer or user. Denote $\left[s_{i, \min }, s_{i, \max }\right]$ as the preferred energy range of the $i$-th $\mathrm{EV}$, with $0 \leq s_{i, \min }<s_{i, \max } \leq s_{i \text {,cap }}$. By such constraints, at each time slot $t$, the available energy for regulation and the energy state should satisfy

$$
\begin{aligned}
& s_{i, \min } \leq m_{i, t} \leq s_{i, \max }, \text { and } \\
& s_{i, \min } \leq s_{i, t} \leq s_{i, \max },
\end{aligned}
$$

with (2) satisfied through the $i$-th EV self charging/discharging activities. Hence, at each time slot $t$, the regulation amounts $x_{i d, t}$ and $x_{i u, t}$ are restricted as follows:

$$
\begin{aligned}
& 0 \leq x_{i d, t} \leq h_{i d, t} \triangleq\left[x_{i, \max }, s_{i, \max }-m_{i, t}\right]^{-} \\
& 0 \leq x_{i u, t} \leq h_{i u, t} \triangleq\left[x_{i, \max }, m_{i, t}-s_{i, \min }\right]^{-} .
\end{aligned}
$$

Assume that the initial energy state $s_{i, 0}$ lies in $\left[s_{i, \min }, s_{i, \max }\right]$.

For each EV, the regulation service gain comes at the cost of battery degradation due to charging and discharging ${ }^{1}$. Denote $C_{i}(\cdot)$ as the degradation cost function of the regulation amount for the $i$-th $\mathrm{EV}$, satisfying $0 \leq C_{i}(\cdot) \leq c_{i, \max }$ and $C_{i}(0)=0$. Since faster charging or discharging, i.e., larger $x_{i d, t}$ or $x_{i u, t}$, has a more detrimental effect on the battery life, we assume $C_{i}(\cdot)$ to be convex, continuous, and non-decreasing. We further assume that each EV imposes an upper limit on the average battery degradation, expressed by $\lim _{T \rightarrow \infty} \frac{1}{T} \sum_{t=0}^{T-1} \mathbb{E}\left[\mathbf{1}_{d, t} C_{i}\left(x_{i d, t}\right)+\mathbf{1}_{u, t} C_{i}\left(x_{i u, t}\right)\right] \leq c_{i, \text { up }}$, with the expectation taken over all randomness in the system.

Finally, the total regulation service provided by the EVs may not be sufficient to serve the requested regulation amount. For brevity, define $x_{i, t} \triangleq \mathbf{1}_{d, t} x_{i d, t}+\mathbf{1}_{u, t} x_{i u, t}$ as the regulation amount allocated to the $i$-th EV at time slot $t$. Such insufficiency means that $\sum_{i=1}^{N} x_{i, t}<\left|G_{t}\right|$ for regulation down or up. This could be due to, for example, a lack of participating EVs, or high cost of battery degradation. The gap between $\sum_{i=1}^{N} x_{i, t}$ and $\left|G_{t}\right|$ represents an energy surplus in the case of regulation down, or an energy deficit in the case of regulation up. Such surplus or deficit must be cleared, or the regulation service fails. Therefore, from time to time, the aggregator may need to exploit more expensive external energy sources, such as from the traditional regulation market. Denote the unit costs of clearing energy surplus and energy deficit at time slot $t$ as $e_{s, t}$ and $e_{d, t}$, respectively, which are both restricted in $\left[e_{\min }, e_{\max }\right]$. Then the total cost for the aggregator at time slot $t$ is

$$
e_{t} \triangleq \mathbf{1}_{d, t} e_{s, t}\left(G_{t}-\sum_{i=1}^{N} x_{i d, t}\right)+\mathbf{1}_{u, t} e_{d, t}\left(\left|G_{t}\right|-\sum_{i=1}^{N} x_{i u, t}\right) \text {. }
$$

\section{B. Fair Regulation Allocation through Welfare Maximization}

The objective of the aggregator is to maximize the longterm social welfare of the aggregator-EVs system, i.e., to fairly allocate the regulation amount among the EVs, while respecting battery degradation constraints of EVs and reducing the need to utilize expensive external energy sources. To this

\footnotetext{
${ }^{1}$ We ignore the energy cost/benefit of regulation up/down service, since the long-term average of the net regulation energy from the power grid is close to zero [16].
} 
end, we have the following stochastic optimization problem:

P1:

$$
\begin{aligned}
\max _{\mathbf{x}_{d, t}, \mathbf{x}_{u, t}} & \sum_{i=1}^{N} \omega_{i} U\left(\lim _{T \rightarrow \infty} \frac{1}{T} \sum_{t=0}^{T-1} \mathbb{E}\left[x_{i, t}\right]\right)-\lim _{T \rightarrow \infty} \frac{1}{T} \sum_{t=0}^{T-1} \mathbb{E}\left[e_{t}\right] \\
\text { s.t. } \quad & 0 \leq x_{i d, t} \leq h_{i d, t}, \forall i \\
& 0 \leq x_{i u, t} \leq h_{i u, t}, \forall i \\
& \sum_{i=1}^{N} x_{i d, t} \leq \mathbf{1}_{d, t} G_{t} \\
& \sum_{i=1}^{N} x_{i u, t} \leq \mathbf{1}_{u, t}\left|G_{t}\right| \\
& \lim _{T \rightarrow \infty} \frac{1}{T} \sum_{t=0}^{T-1} \mathbb{E}\left[\mathbf{1}_{d, t} C_{i}\left(x_{i d, t}\right)+\mathbf{1}_{u, t} C_{i}\left(x_{i u, t}\right)\right] \leq c_{i, \text { up }}, \forall i,
\end{aligned}
$$

where $U(\cdot)$ is a utility function assumed to be concave, continuous, and non-decreasing, with a domain bounded within $\left[0, x_{i, \max }\right], \forall i$, and $\omega_{i}>0$ is the normalized weight associated with the $i$-th EV. Furthermore, to facilitate later analysis, we assume that $U(\cdot)$ satisfies

$$
U(x) \leq U(0)+\mu x, \forall x \in\left[0,\left[x_{1, \max }, \cdots, x_{N, \max }\right]^{+}\right],
$$

where $\mu>0$. One sufficient condition for (9) to hold is that $U(\cdot)$ has finite positive derivate at zero, such as $U(x)=$ $\log (1+x)$. Denote the system state at time slot $t$ as

$$
A_{t} \triangleq\left(G_{t}, \mathbf{a}_{t}, e_{s, t}, e_{d, t}\right) .
$$

The expectations in $\mathbf{P 1}$ are taken over $A_{t}$. From (6) and (7), if $G_{t}>0$ then $x_{i u, t}=0, \forall i$ and if $G_{t}<0$ then $x_{i d, t}=0, \forall i$.

\section{Welfare-MaXimizing Regulation Allocation}

To solve P1, we propose a real-time welfare-maximizing regulation allocation (WMRA) algorithm by adopting a general framework of Lyapunov optimization. We apply a sequence of two reformulations to the original problem, leading to a solution that is asymptotically close to optimal as the EV battery capacity increases.

\section{A. Problem Transformation}

The objective of $\mathbf{P 1}$ contains a function of long-term average, which complicates the problem. However, in general, such a problem can be converted to a problem of maximizing a long-term average of the function [14]. Specifically, we transform $\mathbf{P 1}$ as follows.

We first introduce an auxiliary $N$-dimensional vector $\mathbf{z}_{t} \triangleq\left[z_{1, t}, \cdots, z_{N, t}\right]$ with the constraints

$$
\begin{aligned}
& 0 \leq z_{i, t} \leq x_{i, \max }, \forall i, \text { and } \\
& \lim _{T \rightarrow \infty} \frac{1}{T} \sum_{t=0}^{T-1} \mathbb{E}\left[z_{i, t}\right]=\lim _{T \rightarrow \infty} \frac{1}{T} \sum_{t=0}^{T-1} \mathbb{E}\left[x_{i, t}\right], \forall i .
\end{aligned}
$$

With the above constraints, the auxiliary variable $z_{i, t}$ and the regulation allocation amount $x_{i, t}$ are within the same range and have the same long-term average behavior. We now consider the following problem.

P2:

$$
\begin{aligned}
\max _{\mathbf{x}_{d, t}, \mathbf{x}_{u, t}, \mathbf{z}_{t}} & \lim _{T \rightarrow \infty} \frac{1}{T} \sum_{t=0}^{T-1} \mathbb{E}\left[\left(\sum_{i=1}^{N} \omega_{i} U\left(z_{i, t}\right)\right)-e_{t}\right] \\
\text { s.t. } & (4),(5),(6),(7),(8),(11), \text { and }(12) .
\end{aligned}
$$

Compared with $\mathbf{P 1}, \mathbf{P 2}$ is optimized over $\mathbf{x}_{d, t}, \mathbf{x}_{u, t}$ and $\mathbf{z}_{t}$, with two more constraints (11) and (12). Note that $\mathbf{P} 2$ contains no function of time average; instead, it maximizes a long-term time average of the expected social welfare.

Denote $\left(\mathbf{x}_{d, t}^{\mathrm{opt}}, \mathbf{x}_{u, t}^{\mathrm{opt}}\right)$ as an optimal solution for P1, and $\left(\mathbf{x}_{d, t}^{*}, \mathbf{x}_{u, t}^{*}, \mathbf{z}_{t}^{*}\right)$ as an optimal solution for P2. Define $\overline{\mathbf{z}}_{t}^{\mathrm{opt}} \triangleq\left[\bar{z}_{1, t}^{\mathrm{opt}}, \cdots, \bar{z}_{N, t}^{\mathrm{opt}}\right]$ with

$$
\bar{z}_{i, t}^{\mathrm{opt}} \triangleq \lim _{T \rightarrow \infty} \frac{1}{T} \sum_{t=0}^{T-1} \mathbb{E}\left[x_{i, t}^{\mathrm{opt}}\right], \quad \forall i .
$$

Denote the objective functions of $\mathbf{P} \mathbf{1}$ and $\mathbf{P} \mathbf{2}$ as $f_{1}(\cdot)$ and $f_{2}(\cdot)$, respectively. The equivalence of $\mathbf{P 1}$ and $\mathbf{P 2}$ is stated below.

Lemma 1: $\mathbf{P 1}$ and $\mathbf{P} 2$ have the same optimal objective, i.e., $f_{1}\left(\mathbf{x}_{d, t}^{\mathrm{opt}}, \mathbf{x}_{u, t}^{\mathrm{opt}}\right)=f_{2}\left(\mathbf{x}_{d, t}^{*}, \mathbf{x}_{u, t}^{*}, \mathbf{z}_{t}^{*}\right)$. Furthermore, $\left(\mathbf{x}_{d, t}^{\mathrm{opt}}, \mathbf{x}_{u, t}^{\mathrm{opt}}, \overline{\mathbf{z}}_{t}^{\mathrm{opt}}\right)$ is an optimal solution for $\mathbf{P} \mathbf{2}$, and $\left(\mathbf{x}_{d, t}^{*}, \mathbf{x}_{u, t}^{*}\right)$ is an optimal solution for $\mathbf{P 1}$.

Proof: The proof follows the general framework given in [14]. Details specific to our system are given in [17].

Lemma 1 indicates that the transformation from P1 to P2 results in no loss of optimality. Thus, in the following, we focus on solving $\mathbf{P 2}$ instead.

\section{B. Problem Relaxation}

P2 is still a challenging problem since in constraints (4) and (5), the regulation allocation amount of each EV depends on its current available energy state $m_{i, t}$, which couples with all previous regulation allocation amounts and self charging/discharging amounts. To avoid such coupling, we relax the constraints of $x_{i d, t}$ and $x_{i u, t}$ and introduce $\mathbf{P 3}$ below.

P3:

$$
\begin{aligned}
\max _{\mathbf{x}_{d, t}, \mathbf{x}_{u, t}, \mathbf{z}_{t}} & \lim _{T \rightarrow \infty} \frac{1}{T} \sum_{t=0}^{T-1} \mathbb{E}\left[\left(\sum_{i=1}^{N} \omega_{i} U\left(z_{i, t}\right)\right)-e_{t}\right] \\
\text { s.t. } \quad & 0 \leq x_{i d, t} \leq x_{i, \max }, \forall i \\
& 0 \leq x_{i u, t} \leq x_{i, \max }, \forall i \\
& \lim _{T \rightarrow \infty} \frac{1}{T} \sum_{t=0}^{T-1} \mathbb{E}\left[a_{i, t}+b_{i, t}\right]=0, \forall i \\
& (6),(7),(8),(11), \text { and }(12) .
\end{aligned}
$$

In P3, we have replaced the constraints (4) and (5) in $\mathbf{P} 2$ with (13), (14), and (15), so have avoided the coupling problem. We now demonstrate that, any $\left(\mathbf{x}_{d, t}, \mathbf{x}_{u, t}\right)$ that is feasible for $\mathbf{P 2}$ is also feasible for P3. In other words, the feasible set of optimization variables is enlarged from $\mathbf{P 2}$ to $\mathbf{P 3}$, and the optimal value of $\mathbf{P 3}$ is greater than that of $\mathbf{P 2}$. 
Since (4) and (5) are equivalent to (3), (13), and (14), it suffices to show (3) implies (15). Summing both sides of (1) over $t \in\{0,1, \cdots, T-1\}$ and dividing them by $T$ leads to

$$
\frac{s_{i, T}}{T}-\frac{s_{i, 0}}{T}=\frac{1}{T} \sum_{t=0}^{T-1}\left[a_{i, t}+b_{i, t}\right] .
$$

Taking expectations over both sides of (16) and taking limit gives

$$
\lim _{T \rightarrow \infty} \frac{\mathbb{E}\left[s_{i, T}\right]}{T}-\lim _{T \rightarrow \infty} \frac{\mathbb{E}\left[s_{i, 0}\right]}{T}=\lim _{T \rightarrow \infty} \frac{1}{T} \sum_{t=0}^{T-1} \mathbb{E}\left[a_{i, t}+b_{i, t}\right] .
$$

Since $s_{i, T}$ and $s_{i, 0}$ are always bounded based on (3), the left hand side equals zero, which leads to (15).

The relaxed problem P3 allows us to apply Lyapunov optimization to design a real-time algorithm for solving welfare maximization. We will show later that in fact our developed solution also satisfies (4) and (5), thus providing an optimal solution for $\mathbf{P 2}$ and the original problem P1. This relaxation technique to accommodate the type of time-coupled action constraints such as (4) and (5) is first introduced in [18] for a power-cost minimization problem in data centers equipped with stored energy. Unlike in [18], the structure of our problem is more complicated, with a nonlinear objective which allows both positive and negative values for the energy requirement $G_{t}$. Thus, the algorithm design is more involved to ensure that the original constraints in $\mathbf{P 2}$ are satisfied.

\section{WMRA Algorithm}

Define three virtual queues for each EV with the associated queue backlogs $H_{i, t}, J_{i, t}$, and $K_{i, t}$ evolving as follows:

$$
\begin{aligned}
& J_{i, t+1}=\left[J_{i, t}+\mathbf{1}_{d, t} C_{i}\left(x_{i d, t}\right)+\mathbf{1}_{u, t} C_{i}\left(x_{i u, t}\right)-c_{i, \text { up }}\right]^{+}, \forall i, \\
& H_{i, t+1}=H_{i, t}+z_{i, t}-x_{i, t}, \forall i \\
& K_{i, t+1}=K_{i, t}+a_{i, t}+b_{i, t}, \forall i .
\end{aligned}
$$

Define $\mathbf{H}_{t} \triangleq\left[H_{1, t}, \cdots, H_{N, t}\right], \mathbf{J}_{t} \triangleq\left[J_{1, t}, \cdots, J_{N, t}\right], \mathbf{K}_{t} \triangleq\left[K_{1, t}\right.$, $\left.\cdots, K_{N, t}\right]$, and $\boldsymbol{\Theta}_{t} \triangleq\left[\mathbf{H}_{t}, \mathbf{J}_{t}, \mathbf{K}_{t}\right]$. Initialize $\mathbf{H}_{0}=\mathbf{J}_{0}=\mathbf{0}$. Note that the queue backlog $K_{i, t}$ and the energy state $s_{i, t}$ have the same evolutionary behavior. We relate them as

$$
K_{i, t}=s_{i, t}-c_{i}
$$

where the constant $c_{i} \triangleq s_{i, \min }+2 x_{i, \max }+d_{i, \max }+V\left(\omega_{i} \mu+\right.$ $\left.e_{\max }\right)$ with $V \in\left[0, V_{\max }\right]$ and

$$
V_{\max } \triangleq \min _{1 \leq i \leq N}\left\{\frac{s_{i, \max }-s_{i, \min }-4 x_{i, \max }-d_{i, \max }}{2\left(\omega_{i} \mu+e_{\max }\right)}\right\} .
$$

Note that $x_{i, \max }$ and $d_{i, \max }$ are generally much smaller than the energy capacity. For example, for Tesla Model S, the energy capacity is $40 \mathrm{kWh}$ and $x_{i, \max }=d_{i, \max }=0.83 \mathrm{kWh}$ if the maximum charging rate is applied and the regulation duration is 5 minutes. Therefore, generally we always have $V_{\max }>0$. The reason why $c_{i}$ is defined in such a specific form will be clear later.

From (17), $J_{i, t}$ is non-negative, and it will increase (resp. decrease) if the current degradation cost is above (resp. below) the upper bound. From (18), $H_{i, t}$ can be positive or negative, and it will increase (resp. decrease) if $z_{i, t}$ is larger (resp. smaller) than $x_{i, t}$. The fluctuation of $K_{i, t}$ can be explained similarly as $H_{i, t}$. By introducing the virtual queues, the constraints (8), (12), and (15) hold if the queues $J_{i, t}, H_{i, t}$, and $K_{i, t}$ are mean rate stable, respectively [14].

Define the Lyapunov function $L\left(\Theta_{t}\right) \triangleq \frac{1}{2} \sum_{i=1}^{N}\left(H_{i, t}^{2}+\right.$ $\left.J_{i, t}^{2}+K_{i, t}^{2}\right)$, and the associated Lyapunov drift as $\Delta\left(\boldsymbol{\Theta}_{t}\right) \triangleq \mathbb{E}\left[L\left(\boldsymbol{\Theta}_{t+1}\right)-L\left(\boldsymbol{\Theta}_{t}\right) \mid \boldsymbol{\Theta}_{t}\right]$. We have the following:

Lemma 2: The Lyapunov drift $\Delta\left(\boldsymbol{\Theta}_{t}\right)$ is upper-bounded as

$$
\begin{aligned}
& \Delta\left(\boldsymbol{\Theta}_{t}\right) \leq B+\sum_{i=1}^{N} H_{i, t} \mathbb{E}\left[z_{i, t}-x_{i, t} \mid \boldsymbol{\Theta}_{t}\right]+\sum_{i=1}^{N} K_{i, t} \mathbb{E}\left[a_{i, t}+b_{i, t}\right. \\
& \left.\mid \boldsymbol{\Theta}_{t}\right]+\sum_{i=1}^{N} J_{i, t} \mathbb{E}\left[\mathbf{1}_{d, t} C_{i}\left(x_{i d, t}\right)+\mathbf{1}_{u, t} C_{i}\left(x_{i u, t}\right)-c_{i, \text { up }} \mid \boldsymbol{\Theta}_{t}\right],
\end{aligned}
$$

with $B \triangleq \frac{1}{2} \sum_{i=1}^{N}\left[x_{i, \max }^{2}+\left(d_{i, \max }+x_{i, \max }\right)^{2}+\left[c_{i, \text { up }}^{2},\left(c_{i, \max }-\right.\right.\right.$ $\left.\left.\left.c_{i, \text { up }}\right)^{2}\right]^{+}\right]$.

Proof: See technical report [17].

Express the drift-plus-penalty function as $\Delta\left(\boldsymbol{\Theta}_{t}\right)+$ $V \mathbb{E}\left[e_{t}-\sum_{i=1}^{N} \omega_{i} U\left(z_{i, t}\right) \mid \Theta_{t}\right]$. From Lemma 2, it is upper bounded as

$$
\begin{aligned}
& \Delta\left(\boldsymbol{\Theta}_{t}\right)+V \mathbb{E}\left[e_{t}-\sum_{i=1}^{N} \omega_{i} U\left(z_{i, t}\right) \mid \boldsymbol{\Theta}_{t}\right] \\
& \leq B+V \mathbb{E}\left[e_{t}-\sum_{i=1}^{N} \omega_{i} U\left(z_{i, t}\right) \mid \boldsymbol{\Theta}_{t}\right] \\
& +\sum_{i=1}^{N} H_{i, t} \mathbb{E}\left[z_{i, t}-x_{i, t} \mid \boldsymbol{\Theta}_{t}\right]+\sum_{i=1}^{N} K_{i, t} \mathbb{E}\left[a_{i, t}+b_{i, t} \mid \boldsymbol{\Theta}_{t}\right] \\
& +\sum_{i=1}^{N} J_{i, t} \mathbb{E}\left[\mathbf{1}_{d, t} C_{i}\left(x_{i d, t}\right)+\mathbf{1}_{u, t} C_{i}\left(x_{i u, t}\right)-c_{i, u p} \mid \boldsymbol{\Theta}_{t}\right],
\end{aligned}
$$

The proposed WMRA algorithm minimizes the right-handside of (22) at each time slot. This is equivalent to solving the following decoupled sub-problems with respect to $\mathbf{z}_{t}, \mathbf{x}_{d, t}$ and $\mathbf{x}_{u, t}$, respectively. Specifically, solve (a) with respect to $z_{i, t}, \forall i$ :

$$
\begin{aligned}
& \text { (a): } \quad \min _{z_{i, t}} H_{i, t} z_{i, t}-\omega_{i} V U\left(z_{i, t}\right) \\
& \text { s.t. } \quad 0 \leq z_{i, t} \leq x_{i, \max } .
\end{aligned}
$$

For $G_{t}>0$, solve

$$
\begin{gathered}
\text { (b1): } \min _{\mathbf{x}_{d, t}} V e_{s, t}\left(G_{t}-\sum_{i=1}^{N} x_{i d, t}\right)-\sum_{i=1}^{N} H_{i, t} x_{i d, t} \\
+\sum_{i=1}^{N} J_{i, t} C_{i}\left(x_{i d, t}\right)+\sum_{i=1}^{N} K_{i, t}\left(a_{i, t}+x_{i d, t}\right) \\
\text { s.t. } \quad 0 \leq x_{i d, t} \leq x_{i, \max }, \sum_{i=1}^{N} x_{i d, t} \leq G_{t} .
\end{gathered}
$$


Algorithm 1 Welfare-Maximizing Regulation Allocation (WMRA) Algorithm.

1: The aggregator initializes the virtual queues as $J_{i, 0}=$ $H_{i, 0}=0$, and $K_{i, 0}=s_{i, 0}-s_{i, \min }-2 x_{i, \max }-d_{i, \max }-$ $V\left(\omega_{i} \mu+e_{\max }\right), \forall i$.

2: At the beginning of each time slot $t$, perform the following steps sequentially.

(2a) Each EV reports its self charging/discharging behavior and the associated amount (if any) to the aggregator.

(2b) The aggregator observes $G_{t}, e_{s, t}, e_{d, t}, \mathbf{J}_{t}, \mathbf{H}_{t}$, and $\mathbf{K}_{t}$.

(2c) The aggregator performs the following tasks: Solve (a) and record an optimal solution $\tilde{\mathbf{z}}_{t}$. If $G_{t}>0$, solve (b1) and record an optimal solution $\tilde{\mathbf{x}}_{d, t}$. If $G_{t}<0$, solve (b2) and record an optimal solution $\tilde{\mathbf{x}}_{u, t}$. Allocate the regulation amounts based on $\tilde{\mathbf{x}}_{d, t}$ and $\tilde{\mathbf{x}}_{u, t}$. If $\sum_{i=1}^{N} \tilde{x}_{i d, t}<G_{t}$ or $\sum_{i=1}^{N} \tilde{x}_{i u, t}<$ $\left|G_{t}\right|$, then clear the imbalance using external energy sources.

(2d) Update the virtual queues $J_{i, t}, H_{i, t}$, and $K_{i, t}, \forall i$, based on (17), (18), and (19), respectively.

For $G_{t}<0$, solve

$$
\begin{gathered}
\text { (b2): } \min _{\mathbf{x}_{u, t}} V e_{d, t}\left(\left|G_{t}\right|-\sum_{i=1}^{N} x_{i u, t}\right)-\sum_{i=1}^{N} H_{i, t} x_{i u, t} \\
+\sum_{i=1}^{N} J_{i, t} C_{i}\left(x_{i u, t}\right)+\sum_{i=1}^{N} K_{i, t}\left(a_{i, t}-x_{i u, t}\right) \\
\text { s.t. } \quad 0 \leq x_{i u, t} \leq x_{i, \max }, \sum_{i=1}^{N} x_{i u, t} \leq\left|G_{t}\right| .
\end{gathered}
$$

Note that (a), (b1), and (b2) are all convex problems, so they can be efficiently solved using standard methods such as the interior point method and the Lagrange dual method. We summarize WMRA in Algorithm 1. From Steps (2c) and (2d), the solutions of (a) and (b1) (or (b2)) affect each other over multiple time slots through the update of $H_{i, t}, \forall i$. Note that, to perform WMRA, no statistical information of the system is needed, which makes it easy to implement.

\section{Performance Analysis}

Denote the allocated regulation amounts under WMRA as $\tilde{\mathbf{x}}_{d, t} \triangleq\left[\tilde{x}_{1 d, t}, \cdots, \tilde{x}_{N d, t}\right]$ and $\tilde{\mathbf{x}}_{u, t} \triangleq\left[\tilde{x}_{1 u, t}, \cdots, \tilde{x}_{N u, t}\right]$. In the following lemma, we characterize sufficient conditions under which the unique optimal solution of $x_{i d, t}$ and $x_{i u, t}$ is zero.

Lemma 3: Under the WMRA algorithm, we have

1) for $G_{t}>0$, if $K_{i, t}>x_{i, \max }+V\left(\omega_{i} \mu+e_{\max }\right)$ then $\tilde{x}_{i d, t}=0$; and

2) for $G_{t}<0$, if $K_{i, t}<-x_{i, \max }-V\left(\omega_{i} \mu+e_{\max }\right)$ then $\tilde{x}_{i u, t}=0$.

Proof: See technical report [17].
Using Lemma 3, we can prove that queue backlog $K_{i, t}$ is lower and upper bounded.

Lemma 4: Under the WMRA algorithm, queue backlog $K_{i, t}$ associated with the $i$-th $\mathrm{EV}$ is bounded by $s_{i, \min }-c_{i} \leq$ $K_{i, t} \leq s_{i, \max }-c_{i}$.

Proof: See technical report [17].

Since $K_{i, t} \triangleq s_{i, t}-c_{i}$, from Lemma 4 there is $s_{i, \min } \leq$ $s_{i, t} \leq s_{i, \max }$, demonstrating that under WMRA, the energy state is bounded within the preferred range for each EV. In the following theorem, we characterize the performance of WMRA with respect to our original problem P1.

Theorem 1: Given that the system state $A_{t}$ in (10) is i.i.d. over time slots, we have

1) $\left(\tilde{\mathbf{x}}_{d, t}, \tilde{\mathbf{x}}_{u, t}\right)$ is feasible for $\mathbf{P 1}$.

2) $f_{1}\left(\tilde{\mathbf{x}}_{d, t}, \tilde{\mathbf{x}}_{u, t}\right) \geq f_{1}\left(\mathbf{x}_{d, t}^{\mathrm{opt}}, \mathbf{x}_{u, t}^{\mathrm{opt}}\right)-\frac{B}{V}$, where $B \triangleq \frac{1}{2} \sum_{i=1}^{N}\left[x_{i, \max }^{2}+\left(d_{i, \max }+x_{i, \max }\right)^{2}\right.$ $\left.+\left[c_{i, \text { up }}^{2},\left(c_{i, \max }-c_{i, \text { up }}\right)^{2}\right]^{+}\right]$and $V \in\left[0, V_{\max }\right]$.

Proof: See technical report [17].

Remarks: Theorem 1 indicates that the welfare performance of WMRA is away from the optimum by $O(1 / V)$. Hence, the larger $V$, the better the performance of WMRA. However, in practical systems, due to the boundedness condition of EVs' battery capacities, $V$ cannot be arbitrarily large and is upper bounded by $V_{\max }$. Note that $V_{\max }$ increases with the smallest span of the EVs' preferred ranges, i.e., $\min _{1 \leq i \leq N}\left\{s_{i, \max }-\right.$ $\left.s_{i, \min }\right\}$. Therefore, roughly speaking, the performance gap between WMRA and the optimum decreases as the smallest battery capacity increases; mathematically, if the EVs' battery capacities go to infinity, WMRA would achieve exactly the optimum.

\section{Simulation Results}

Although WMRA is shown to be asymptotically optimal in the previous section, we are further interested in its performance in example numerical settings. Towards this goal, we have simulated an aggregator-EVs system with parameters drawn from practical scenarios. We compare the performance of WMRA with that of a suboptimal greedy algorithm.

We assume that the aggregator is connected with $N=100$ EVs, evenly split into Type I (Ford Focus Electric) and Type II (Tesla Model S). The parameters of Type I and Type II EVs are summarized in Table I. For simplicity, assume that the EVs do not perform self charging/discharging. The regulation interval $\Delta t=5$ minutes, and the regulation energy amount $G_{t}$ is drawn uniformly from $[-69.2,69.2](\mathrm{kWh})$ at each time slot, with $69.2 \mathrm{kWh}$ being the maximum total allowed energy amount from all EVs at each time slot. The unit costs of external sources, i.e., $e_{s, t}$ and $e_{d, t}$, are drawn uniformly from $[0.1,0.12]$ (dollars/kWh). In the objective function of $\mathbf{P 1}$, we set $U(x)=\log (1+x)$ and $\omega_{i}=1, \forall i$. The battery degradation cost function of each EV is $C_{i}(x)=x^{2}$, and the upper bound $c_{i, \text { up }}$ is set to be $x_{i, \max }^{2} / 4$.

To allocate the requested regulation amount, we apply WMRA at each time slot with $V=V_{\max }$. The simulation is performed over $T=1000$ time slots. For comparison, we 
TABLE I

PARAMETERS FOR TYPE I AND TYPE II EVS

\begin{tabular}{l|l|l}
\hline & Type I EV & Type II EV \\
\hline$s_{i, \mathrm{cap}}(\mathrm{kWh})$ & 23 & 40 \\
\hline$x_{i, \max }(\mathrm{kWh})$ & 0.55 & 0.83 \\
\hline
\end{tabular}

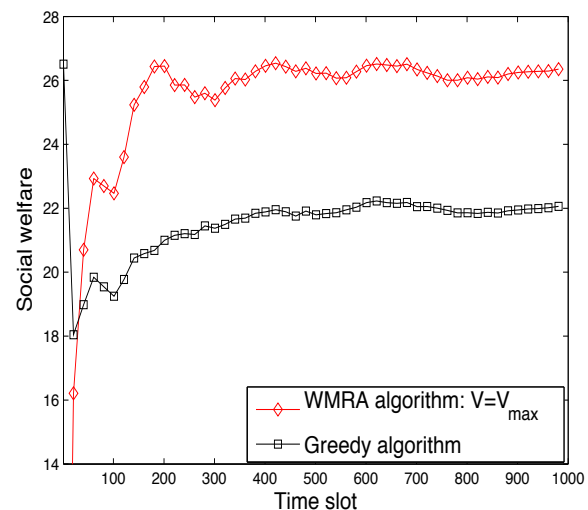

Fig. 1. Time-averaged social welfare with $s_{i, \min }=0.1 s_{i, \text { cap }}$ and $s_{i, \max }=$ $0.9 s_{i, \text { cap. }}$

consider a greedy algorithm, which only optimizes the system performance at the current time slot. Thus, the regulation allocation at each time slot is derived from the following optimization problem.

$$
\begin{aligned}
\max _{\mathbf{x}_{d, t}, \mathbf{x}_{u, t}} & \left(\sum_{i=1}^{N} \omega_{i} U\left(x_{i, t}\right)\right)-e_{t} \\
\text { s.t. } & (4),(5),(6),(7), \text { and } \\
& \mathbf{1}_{d, t} C_{i}\left(x_{i d, t}\right)+\mathbf{1}_{u, t} C_{i}\left(x_{i u, t}\right) \leq c_{i, \text { up }}, \forall i .
\end{aligned}
$$

The above is a convex optimization problem, which can be solved by standard numerical algorithms.

We compare the performance of WMRA and the greedy algorithm in Figs. 1 and 2. In Fig. 1, for $s_{i, \text { min }}=0.1 s_{i \text {,cap }}$ and $s_{i, \text { max }}=0.9 s_{i, \text { cap }}$, WMRA is uniformly superior to the greedy algorithm by about $20 \%$ after 100 time slots. In Fig. 2, we set $s_{i, \text { min }}=0.1 s_{i, \text { cap }}$ and vary $s_{i, \text { max }}$ from $0.3 s_{i \text {,cap }}$ to $0.9 s_{i, \text { cap }}$. The observations are, first, WMRA uniformly outperforms the greedy algorithm; second, as $s_{i, \max }$ increases, the social welfare under WMRA keeps on increasing while that under the greedy algorithm reaches saturation when $s_{i, \max } \geq 0.6 s_{i, \text { cap }}$.

\section{CONCLUSION}

We have studied a practical model of an aggregator-EVs system providing regulation service, which accounts for random system dynamics, EV self charging/discharging, battery constraints, and the costs of battery degradation and external energy sources. Adopting a general Lyapunov optimization framework, we have developed a real-time WMRA algorithm for the aggregator to fairly allocate the regulation amount among EVs. The algorithm is proven to be asymptotically optimal, and it offers substantial performance gains over a greedy algorithm.

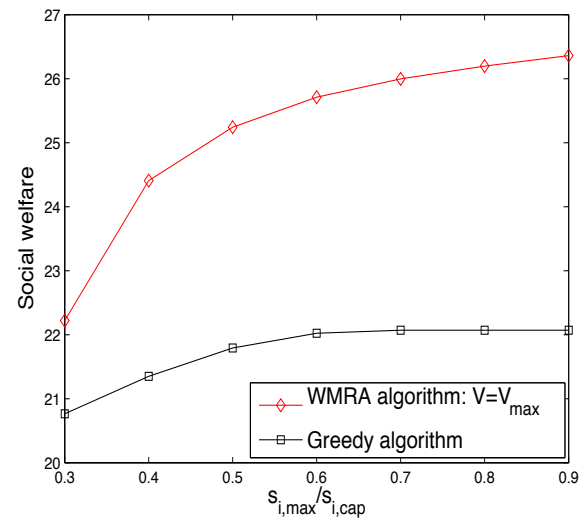

Fig. 2. Time-averaged social welfare with $s_{i, \min }=0.1 s_{i \text {,cap }}$, and various $s_{i, \max }$

\section{REFERENCES}

[1] U.S. Dept. Energy, "One million electric vehicles by 2015," Tech. Rep., Feb. 2011.

[2] C. Guille and G. Gross, "A conceptual framework for the vehicle-togrid (V2G) implementation,” Energy Policy, vol. 37, pp. 4379-4390, Nov. 2009.

[3] J. Lopes, F. Soares, and P. Almeida, "Integration of electric vehicles in the electric power system," Proc. IEEE, vol. 99, pp. 168-183, Jan. 2011

[4] W. Kempton and J. Tomic, "Vehicle-to-grid power fundamentals: calculating capacity and net revenue," J. Power Sources, vol. 144, pp. 268 279, Jun. 2005.

[5] B. Kirby, "Frequency regulation basics and trends," U.S. Dept. Energy, Tech. Rep., 2005.

[6] W. Kempton, V. Udo, K. Huber, K. Komara, S. Letendre, S. Baker, D. Brunner, and N. Pearre, "A test of vehicle-to-grid (V2G) for energy storage and frequency regulation in the PJM system," Tech. Rep., Nov. 2008. [Online]. Available: http://www.udel.edu/V2G/resources/ test-v2g-in-pjm-jan09.pdf

[7] R. Bessa and M. Matos, "Economic and technical management of an aggregation agent for electric vehicles: a literature survey," Eur. Trans. Elect. Power, vol. 22, pp. 334-350, Apr. 2011.

[8] J. Garzas, A. Armada, and G. Granados, "Fair design of plug-in electric vehicles aggregator for V2G regulation," IEEE Trans. Veh. Technol., vol. 61, pp. 3406-3419, oct. 2012.

[9] S. Han, S. Han, and K. Sezaki, "Optimal control of the plug-in electric vehicles for V2G frequency regulation using quadratic programming," in Proc. IEEE ISGT, Jan. 2011.

[10] E. Sortomme and M. Sharkawi, "Optimal scheduling of vehicle-to-grid energy and ancillary services," IEEE Trans. Smart Grid, vol. 3, pp. 351359, Mar. 2012.

[11] S. Han, S. Han, and K. Sezaki, "Development of an optimal vehicleto-grid aggregator for frequency regulation," IEEE Trans. Smart Grid, vol. 1, pp. 65-72, Jun. 2010.

[12] W. Shi and V. Wong, "Real-time vehicle-to-grid control algorithm under price uncertainty," in Proc. IEEE SmartGridComm, Oct. 2011.

[13] C. Wu, H. Rad, and J. Huang, "Vehicle-to-aggregator interaction game," IEEE Trans. Smart Grid, vol. 3, pp. 434-441, Mar. 2012.

[14] M. Neely, Stochastic network optimization with application to communication and queueing systems. Morgan \& Claypool, 2010.

[15] S. Han, S. Han, and K. Sezaki, "Economic assessment on V2G frequency regulation regarding the battery degradation," in Proc. IEEE ISGT, Jan. 2012.

[16] A. Brooks, "Vehicle-to-grid demonstration project: grid regulation ancillary service with a battery electric vehicle," AC Propulsion, Inc., Tech. Rep., 2002.

[17] S. Sun, M. Dong, and B. Liang, "Real-time welfare-maximizing regulation allocation in aggregator-EVs systems," University of Toronto, Tech. Rep., 2013. [Online]. Available: http://www.comm. utoronto.ca/ liang/publications/techreport/techreport_WMRA.pdf

[18] R. Urgaonkar, B. Urgaonkar, M. Neely, and A. Sivasubramaniam, "Optimal power cost management using stored energy in data centers," in Proc. ACM SIGMETRICS, 2011. 